\title{
Analysis of Magnetotactic Behavior by Swimming Assay
}

\author{
Shoutaro SAKaguchi, ${ }^{1}$ Azuma TAOKA, ${ }^{2}$ and Yoshihiro FuKUmori ${ }^{2, \dagger}$ \\ ${ }^{1}$ Department of Life Sciences, Graduate School of Natural Science and Technology, Kanazawa University, \\ Kakuma-machi, Kanazawa 920-1192, Japan \\ ${ }^{2}$ School of Natural Systems, College of Science and Engineering, Kanazawa University, \\ Kakuma-machi, Kanazawa 920-1192, Japan
}

Received November 20, 2012; Accepted February 19, 2013; Online Publication, May 7, 2013

[doi:10.1271/bbb.120880]

Prokaryotic organelles called magnetosomes allow magnetotactic bacteria to navigate along geomagnetic field lines. In this study, we modified a swimming assay commonly used to assess bacterial motility to develop a new method of assessing magnetotactic motility. By this method, the swimming assay was performed in an artificial magnetic field. Magnetotactic bacteria formed a wedge-shaped swimming halo that elongated parallel to the magnetic field. Magnetotactic motility was qualitatively assessed by comparing halo shapes. We termed this method the magnetic swimming assay. On the magnetic swimming assay, the mamK deletion strain formed a shorter halo than the wild type, indicating that the assay sensitively detects differences in magnetotactic motility. Moreover, we isolated two spontaneous magnetotactic motility mutants using magnetic swimming plates. Our findings indicate that the magnetic swimming assay is a useful method for the sensitive analysis of magnetotaxis phenotypes and mutant screening.

Key words: magnetotactic bacteria; motility; swimming; cytoskeleton; magnetosome

Magnetotactic bacteria synthesize unique prokaryotic organelles called magnetosomes, which function as cellular compasses for navigation along Earth's magnetic field. ${ }^{1-5)}$ Magnetosomes have a well-ordered chain-like structure, comprising membrane-enveloped nano-sized magnetic crystals and various specifically associated proteins. Magnetotactic bacteria are generally found at the oxic-anoxic transition zone in aquatic habitats, and magnetotaxis is thought to guide cells downward to less oxygenated regions in aquatic habitats. ${ }^{6,7)}$

Several methods of measuring cellular magnetism in analyzing the molecular mechanisms underlying the magnetotaxis of magnetotactic bacteria have been reported. The simple methods have been developed using microscopic observation and the image analysis techniques to assay swimming behavior and the magnetic responses of individual living cells. ${ }^{8-10)}$ Although these methods are easy to apply, they cannot be used to measure cell magnetism quantitatively. Magnetic moments have also been determined in cells by light-scattering and birefringence techniques, as reported by Rosenblatt et al. ${ }^{11,12)}$ Schüler et al. devel- oped a light-scattering method to measure magnetism quantitatively, and this is now generally accepted as the standard method of testing the magnetism of magnetotactic bacteria. ${ }^{13)}$ They defined $C_{m a g}$ as the ratio of the value of light-scattering in the direction of the magnetic field parallel to the light path to that perpendicular to the light path. This is a fast and sensitive method of monitoring magnetite formation in growing cell cultures. Based on the same principle, Zhao et al. developed a simple measuring apparatus to be used with a spectrophotometer ${ }^{14)}$ (Supplemental Fig. S1; see Biosci. Biotechnol. Biochem. Web site).

Recent progress in molecular biological studies of magnetotactic bacteria have revealed that several tens of magnetosome-associated proteins are encoded in a large genetic island, referred to as the magnetosome island, that is essential for the synthesis of magnetosomes. ${ }^{15-17)}$ These proteins are thought to function in magnetite biomineralization, magnetic sensing, the formation of magnetosome vesicle, and the construction of magnetosomal structures. Although the details of the functions of individual proteins are unclear, comprehensive analysis of the genes encoded in the magnetosome island have provided clues to the mechanisms of magnetosome biogenesis, maintenance, and magnetoreception. ${ }^{18-20)}$ Characterizations of deletion mutants of the individual genes in the magnetosome island have elucidated individual magnetosome-associated protein functions. For example, the MamJ protein is implicated in the control of magnetosome chain assembly. The mamJ deletion mutant shows no change in the synthesis of magnetite crystals, but does not to produce a straight magnetosome chain. ${ }^{21,22)}$ Four small proteins, MamG, MamF, MamD, and MamC, control the grain size of magnetite crystals. Transmission electron microscopy (TEM) of mamGFDC mutant cells revealed the presence of small magnetosome crystals of a cuboidal shape that were aligned in irregular, widely spaced chains. ${ }^{23)}$ Thus, the functions of some magnetosome-associated proteins have been clarified.

The mam $A B$ operon, which encodes 18 genes, is highly conserved in several magnetotactic bacteria species, and is essential for magnetosome formation. ${ }^{15,17)}$ Murat et al. generated individual single-gene deletions of the mamAB operon in Magnetospirillum

$\dagger$ To whom correspondence should be addressed. Tel: +81-76-264-6231; Fax: +81-76-264-6230; E-mail: fukumor@staff.kanazawa-u.ac.jp

Abbreviations: WT, Magnetospillum magneticum AMB-1 wild type; $\Delta$ mamK strain, Magnetospillum magneticum AMB-1 mamK deletion strain; SDS-PAGE, sodium dodecyl sulfate-polyacrylamide gel electrophoresis; PBS, phosphate buffered saline; EMSGM, enriched magnetic spirillum growth medium; TEM, transmission electron microscope 
magneticum AMB-1. ${ }^{24)}$ They measured the $C_{m a g}$ values of these mutants. Although the $C_{m a g}$ values were diminished compared with the wild type in most of the mutants, the $C_{\text {mag }}$ values did not show clear differences between individual mutants. Thus the light-scattering method is powerful tool to assess cellular magnetism, but it is not enough to analyze or compare the phenotypes of magnetosome associated gene mutants.

The term "magnetotaxis" is used to describe the orientation and migration of bacteria along geomagnetic field lines. ${ }^{25}$ ) Magnetotaxis is thought to separate two processes. The first is magnetic orientation (magnetism). Magnetosomes sense a magnetic field, and the magnetic torque is transferred to the cell body. Then the cell orientates to the magnetic field. The second is migration. The cell migrates to a magnetic pole by flagellar rotation along the magnetic field line. Hence the method of measuring magnetotaxis should be assessed in terms of magnetism and motility. Although the light-scattering method assesses the magnetism of cells, it does not assess motility. Indeed, the $C_{m a g}$ value of killed cells (non-motile) is same as that of living cells (motile). Hence this method does not assess in its entirety. A method is needed to assessing both magnetism and motility to investigate magnetotactic behavior.

To establish a new magnetotaxis assay, we improved a bacterial swimming assay that assesses bacterial motility. By this assay, we performed a swimming assay in an artificial magnetic field. The magnetotaxis phenotype was represented as the length of the swimming halo. We found that this method was also applicable to the screening magnetotaxis mutants.

\section{Materials and Methods}

Bacterial strains and culture conditions. The strains and plasmids used in this study are listed in Table 1 . The M. magneticum AMB-1 (ATCC 700264) wild type (WT) and the mamK deletion strain ( $\triangle m a m K$ strain $)^{26)}$ were cultivated in a chemically defined liquid medium, MS-1 medium, ${ }^{27)}$ under an $\mathrm{O}_{2}(1 \%)-\mathrm{N}_{2}(99 \%)$ atmosphere at $28^{\circ} \mathrm{C}$ in the dark.

The $C_{m a g}$ value was measured as described Zhao et al. ${ }^{14)}$ Magnet coils were attached around the cuvette holder in spectrophotometer V-630 UV-VIS (Jasco, Tokyo, Japan). When the current applied to the coils was $5 \mathrm{~A}$, the magnetic field around the cuvette was about $4.25 \mathrm{mT}$. The maximum and minimum optical densities of the cell cultures were generated by direction-changing magnetic fields. The maximum optical density of the cell cultures $\left(\mathrm{OD}_{\max }\right)$ was generated by a magnetic field parallel to the light path. The minimum optical density of the cell cultures $\left(\mathrm{OD}_{\min }\right)$ was generated by a magnetic field perpendicular to the light path. Optical density was measured a wavelength of the $600 \mathrm{~nm}$. The $C_{m a g}$ value was defined as $\left(\mathrm{OD}_{\max } / \mathrm{OD}_{\min }\right)-1^{13)}$ (Supplemental Fig. S1).

Escherichia coli strain XL-1 Blue $\mathrm{MRF}^{28)}$ was used as host for cloning. E. coli strain WM3064 ${ }^{29)}$ was used as donor for the transformation of WT and the $\Delta m a m K$ strain by conjugation. $E$. coli cultures were incubated in Luria-Bertani medium at $37^{\circ} \mathrm{C}$. Antibiotics, including gentamycin and kanamycin sulfate, were added at a final concentration of $5 \mu \mathrm{g} / \mathrm{mL}$ for the AMB-1 strains, and $10 \mu \mathrm{g} / \mathrm{mL}$ and $20 \mu \mathrm{g} / \mathrm{mL}$ for the $E$. coli strains. 2,6-Diaminopimelic acid was added to the medium to a final concentration of $1 \mathrm{mM}$ for $E$. coli strain WM3064.

Cultivation of nonmagnetic M. magneticum AMB-1 WT was carried out under aerobic conditions in 500-mL Sakaguchi flasks containing $200 \mathrm{~mL}$ of MS-1 medium. For aerobic cultivation, cells were incubated in free gas exchange with air at $28^{\circ} \mathrm{C}$, and were agitated at $120 \mathrm{rpm}$ for $48 \mathrm{~h}$ in an incubator shaker. ${ }^{30)}$

Complementation of the mamK deletion strain. The ptac11containing tac promoter ${ }^{31)}$ was digested with HindIII and EcoRI, and the purified tac promoter was subcloned into pBlue script II vector. ${ }^{32)}$ The tac promoter was digested with $\mathrm{XhoI}$ and EcoRI and ligated with broad-host-range plasmid pJN105 ${ }^{33}$ ) to yield plasmid pBBR-tac, which served as negative control. The mamK fragment was amplified by polymerase chain reaction from $M$. magneticum AMB-1 genomic DNA using primer sets mam $K$-F-EcoRI (GGAATTCCAATTGGTCATTTAGCACGCG) and mamK-R-BamHI (CGGGATCCTATCCCCGTCCCGCATATCG). The mamK fragment was excised with EcoRI and BamHI and cloned into the corresponding sites of the pBBR-tac vector, yielding plasmid pBBR-tac-mamK. Complementary plasmid pBBR-tac-mamK and negative control plasmid pBBR-tac were introduced into recipient strain $M$. magneticum AMB-1 of the WT and $\triangle m a m K$ strains by bacterial conjugation with E. coli WM3064 as donor, as previously described. ${ }^{34)}$

Immunoblotting and immunolocalization analyses. Whole-cell extracts of the M. magneticum AMB-1 strains were prepared by boiling the cells in sodium dodecyl sulfate-polyacrylamide gel electrophoresis (SDS-PAGE) sample buffer (2\% SDS, 2\% mercaptoethanol, and $50 \mathrm{~mm}$ Tris- $\mathrm{HCl}, \mathrm{pH} 6.8$ ) for $10 \mathrm{~min}$, and centrifuged at $8,000 \times g$ for $10 \mathrm{~min}$. The samples separated by SDS-PAGE were transferred to polyvinylidene fluoride membranes (Hybond-P, GE Healthcare, Bucks, England) using an electroblotting method at $1 \mathrm{~mA} / \mathrm{cm}^{2}$ for $2 \mathrm{~h}$. Immunoreactive species of anti-MamK antibodies were detected at a dilution of 1:50,000. Goat anti-rabbit IgG conjugated to horseradish peroxidase (GE Healthcare) was used at a dilution of 1:10,000 for ECL Plus Western blotting detecting reagents (GE Healthcare). Chemi-

Table 1. Bacterial Strains and Plasmids Used in This Study

\begin{tabular}{|c|c|c|}
\hline Strains and plasmids & Genotype and makers & Source or reference \\
\hline \multicolumn{3}{|l|}{ M. magneticum } \\
\hline WT & Wild-type strain & ATCC700264 \\
\hline$\Delta m a m K$ & $\operatorname{mamK}$ deletion derivative of WT & 25 \\
\hline WT pBBR-tac & Insertion of control vector (pBBR-tac) in wild type, $\mathrm{Gm}^{\mathrm{r}}$ & This study \\
\hline WT pBBR-tac-mamK & Insertion of $\operatorname{mamK}(\mathrm{pBBR}-\mathrm{tac}-\operatorname{mamK})$ in wild type, $\mathrm{Gm}^{\mathrm{r}}$ & This study \\
\hline$\Delta m a m K$ pBBR-tac & Insertion of control vector (pBBR-tac) in mamK deletion mutants, $\mathrm{Gm}^{\mathrm{r}}$ & This study \\
\hline$\Delta m a m K$ pBBR-tac-mamK & $\begin{array}{l}\text { Complementation of } \operatorname{mamK}(\mathrm{pBBR} \text {-tac-mamK) in } \operatorname{mamK} \text { deletion } \\
\text { mutants, } \mathrm{Gm}^{\mathrm{r}}\end{array}$ & This study \\
\hline \multicolumn{3}{|l|}{ E. coli } \\
\hline XL-1 blue $\mathrm{MRF}^{\prime}$ & $\begin{array}{l}\Delta(m c r A) 183 \Delta\left(m c r \mathrm{CB}^{-} \text {hs } \mathrm{SMR}-m r r\right) 173 \text { endA1 supE44 thi-1 recA1 } \\
\text { gyrA96 relA1 lac }\left[\mathrm{F}^{\prime}, \text { proAB, laqI }{ }^{\mathrm{q} Z} \text { lacZ } \Delta \mathrm{M} 15, \text { Tn10 }\left(\text { Tet }^{\mathrm{r}}\right)\right.\end{array}$ & 27 \\
\hline WM3064 & $\begin{array}{l}\text { thrB1004 pro thi rpsL lacZ } \triangle M 15 R P 4-1360 \Delta(\text { araBAD) } 567 \\
\Delta \text { dapA1341::[erm } \operatorname{pir}(w t)]\end{array}$ & 28 \\
\hline \multicolumn{3}{|l|}{ Plasmids } \\
\hline Ptac11 & tac promoter coding vector, $A m p^{r}$ & 30 \\
\hline pJN105 & Brodhost range vectorcoding araC, $\mathrm{BAD}$ promoter, $\mathrm{Gm}^{\mathrm{r}}$ & 32 \\
\hline pBBR-tac & pJN105 with tac promoter inserting into BAD promoter & This study \\
\hline pBBR-tac-mamK & pBBR-tac with mamK fragment inserting into MCS & This study \\
\hline
\end{tabular}


fluoresence data were collected using a luminescent image analyzer LAS 3000 (Fujifilm, Tokyo, Japan). The band intensities were quantified using Multi Gauge version 2.2 software (Fujifilm).

For immunolocalization of MamK filaments, cells were grown for $48 \mathrm{~h}$ to the early stationary phase, fixed in $2 \%$ formaldehyde in phosphate buffered saline (PBS; $140 \mathrm{~mm} \mathrm{NaCl}, 3 \mathrm{~mm} \mathrm{KCl}, 8 \mathrm{~mm}$ $\mathrm{Na}_{2} \mathrm{HPO}_{4}$, and $1.5 \mathrm{~mm} \mathrm{KH} \mathrm{KO}_{4}$ ) for $10 \mathrm{~min}$ at $25^{\circ} \mathrm{C}$. The cells were washed 2 times with PBS and suspended in sterile distilled water. They were treated with varying amounts of lysozyme $(5-50 \mathrm{mg} / \mathrm{mL})$ and adhered to the glass slides and dried completely. The slides were immersed in PBS, $0.5 \%$ bovine serum albumin for $10 \mathrm{~min}$ and then incubated with primary anti-MamK antibody at a dilution of 1:100 in the same buffer for $30 \mathrm{~min}$. The slides were washed two times in PBS and incubated with secondary fluorescein isothiocyanate-conjugated anti-rabbit immunoglobulin G (EY Laboratories, California, USA) at a dilution of $1: 1500$

Fluorescence microscopy images were observed with Eclipse E600 (Nikon, Tokyo, Japan). Images were acquired with a DS-Qi1Mc monochrome quantitative digital camera (Nikon) and processed with NIS-Elements software (Nikon) and/or Adobe Photoshop CS2 (Adobe, San Jose, California).

Magnetic swimming assays. Magnetic swimming assays were done by inoculating cells into a semi-solid $0.28 \%$ (weight/volume) enriched magnetic spirillum growth medium (EMSGM) ${ }^{35)}$ agar plate. To demonstrate magnetotaxis, a neodymium magnet $(15 \times 15 \times 1.5 \mathrm{~mm}$, magnetic flux density $=180 \mathrm{mT}$ ) was attached to the edge opposite the inoculation site on the swim plate. The swim plate was put in a custom-made desiccator $(210 \times 240 \times 340 \mathrm{~mm})$. To maintain microaerobic conditions of the desiccator, $\mathrm{O}_{2}(1 \%)-\mathrm{N}_{2}(99 \%)$ gas was allowed to flow in the desiccator at a flow rate $5 \mathrm{~L} / \mathrm{min}$ for $30 \mathrm{~min}$. Then the swim plate was incubated at $25^{\circ} \mathrm{C}$ for $7 \mathrm{~d}$.

To determine the number of magnetosomes per cell in the magnetic swimming assay, the swim plates were divided at 4 points with a $2 \mathrm{~cm}$ between the inoculated cell site and the magnet. The samples were removed at each point on the cultures with a Pasteur pipette and fixed in $2 \%$ formaldehyde in PBS for $10 \mathrm{~min}$ at $25^{\circ} \mathrm{C}$. The cells were washed 2 times with PBS and suspended in sterile distilled water. The samples were observed by TEM, and the mean number of magnetite crystals per cell was counted in at least 50 cells.

Spontaneous magnetotaxis mutants were identified by screening using the swim plate. WT cells were inoculated onto the plate and incubated under microaerobic conditions at $28^{\circ} \mathrm{C}$ for $7 \mathrm{~d}$. The area between point 1 and point 2 of the swimming halo was transferred to liquid MS-1 medium and incubated under microaerobic conditions at $28^{\circ} \mathrm{C}$ in the dark. The culture was inoculated onto a new swim plate and incubated under microaerobic conditions at $28^{\circ} \mathrm{C}$ for $7 \mathrm{~d}$. These screening manipulations were repeated until a different swimming halo was observed as compared to the WT. The cell samples were observed by TEM.

To determine the function of MamK, WT, $\Delta$ mamK, and mamK complementation strains were inoculated on swim plates at various iron concentrations. EMSGM was supplemented with $5 \mathrm{~mL}$ of Wolfe's mineral solution (containing $0.1 \mathrm{~g}$ of $\mathrm{FeSO}_{4} \cdot 7 \mathrm{H}_{2} \mathrm{O}$ in $1,000 \mathrm{~mL}$ water at an iron concentration $0.36 \mathrm{~mm}), 2 \mathrm{~mL}$ of ferric quinate solution (containing $0.27 \mathrm{~g}$ of $\mathrm{FeCl}_{3}$ in $100 \mathrm{~mL}$ of water, at an iron concentration $16.6 \mathrm{mM})$ at a final iron concentration of $35 \mu \mathrm{M} .{ }^{35)}$ The iron concentration of Wolfe's mineral solution and ferric quinate solution (no changes to the other chemical compounds) were modified. An iron-free semi-solid $0.28 \%$ EMSGM agar plate was supplemented with various concentrations of iron using $5 \mathrm{~mL}$ of modified Wolfe's mineral solution and $2 \mathrm{~mL}$ of modified ferric quinate solution at final iron concentrations of $35 \mu \mathrm{M}, 18 \mu \mathrm{M}$, and $9 \mu \mathrm{M}$. The mean length of the swimming halo was scored as the length of the swimming halo for each strain $(\mathrm{mm})$ divided by the distance of the inoculated cell site from the magnet (mm).

Microscopic observation of swimming speeds in liquid cultures. Microscopic observation of the swimming of the strains in liquid cultures was done using an Olympus BH-2 (Olympus, Tokyo, Japan) microscope and an Olympus DP12 camera system (Olympus). Recordings were made using a Leica IM50 version 1.20 system (Olympus), and were analyzed using a Motic Image Plus 2.2s (Shimazu Rika, Tokyo, Japan).
To assay swimming speed, cultures of the WT, the $\Delta \operatorname{mam} K$, and the mamK complementation strain were grown on MS-1 medium under an $\mathrm{O}_{2}(1 \%)-\mathrm{N}_{2}(99 \%)$ atmosphere at $28^{\circ} \mathrm{C}$ in the dark for $7 \mathrm{~d}$. Then $4 \mu \mathrm{L}$ of each culture was dropped onto a slide glass and sealed with a cover glass. The swimming of each strain was observed immediately by dark-field microscopy, and the swimming speeds were calculated as the length of the swimming trajectory of individual cells during a $1 \mathrm{~s}$ exposure time. All results shown are mean swimming speeds of 50 cells.

Electron microscopic observation of the number of magnetite crystals per cell in liquid cultures. WT, $\Delta m a m K$, and mamK complementation strains were inoculated in liquid EMSGM at various concentrations of iron $(35 \mu \mathrm{M}, 18 \mu \mathrm{M}$, and $9 \mu \mathrm{M})$ under an $\mathrm{O}_{2}(1 \%)-\mathrm{N}_{2}$ (99\%) atmosphere at $25^{\circ} \mathrm{C}$ in the dark. These cultures were grown for $24 \mathrm{~h}$ to mid-log phase or $48 \mathrm{~h}$ to early stationary phase. The cell cultures were observed by TEM, and the number of magnetite crystals per cell was counted for at least 50 cells.

Electron microscopy. Cells were adsorbed on carbon-coated copper grids. The samples were viewed and recorded with a JEOL JEM 2000EX transmission electron microscope (JEOL, Tokyo, Japan) at an accelerating voltage of $120 \mathrm{kV}$ in bright-field mode. Some of the grids were negatively stained with $2 \%$ (weight/volume) uranyl acetate for several seconds.

\section{Results and Discussion}

\section{Development of the magnetic swimming assay}

The bacterial swimming assay is a simple method of assessing bacterial chemotaxis and motility. ${ }^{36-38)}$ In this assay, bacterial cells were inoculated onto a semi-solid medium with $0.2-0.5 \%$ (weight/volume) agar. After incubation for several days, the bacteria formed a circular swimming halo. The swimming halo was extended by bacterial flagellar motility. The swimming assay allows for visualization of bacterial motility based on the diameter of the swimming halo. This assay is a commonly used method of assessing the bacterial motility of several species, including E. coli, ${ }^{39)}$ Bacillus subtillis, ${ }^{40)}$ Vibrio parahaemolyticus, ${ }^{41)}$ and Pseudomonas aeruginosa. ${ }^{42)}$

In the present study, we modified the swimming assay to assess magnetotaxis qualitatively. Figure 1a shows the swim plate after $7 \mathrm{~d}$ of incubation. In the magnetized plates, a wedge-shaped halo formed from the inoculation site to the south pole of neodymium magnet (Fig. 1a). In the nonmagnetized plates, however, the cells formed a circular swimming halo (Fig. 1b). When the nonmagnetic M. magneticum AMB-1 cells were inoculated onto the swim plate, a circular halo formed in the magnetic field (Fig. 1c). Hence we concluded that the wedgeshaped halo demonstrated the magnetic motile property of the magnetotactic bacteria. We labeled this method the magnetic swimming assay.

Why did the magnetotactic bacteria form a wedgeshaped halo? The magnetic field lines are concentrated into a pole of the neodymium magnet. Magnetotactic bacteria migrated in agar medium along the magnetic field to the magnetic pole. Thus the magnetotactic bacteria formed a wedge-shaped halo in the swim plate.

We examined to determine whether the magnetosomes of the cells differed depending on the position of the swimming halo. Figure 1e shows the sampling points where we harvested the cells. The distance between sampling points was $2 \mathrm{~cm}$. The cells were removed, and fixed in $2 \%$ formaldehyde in PBS for 

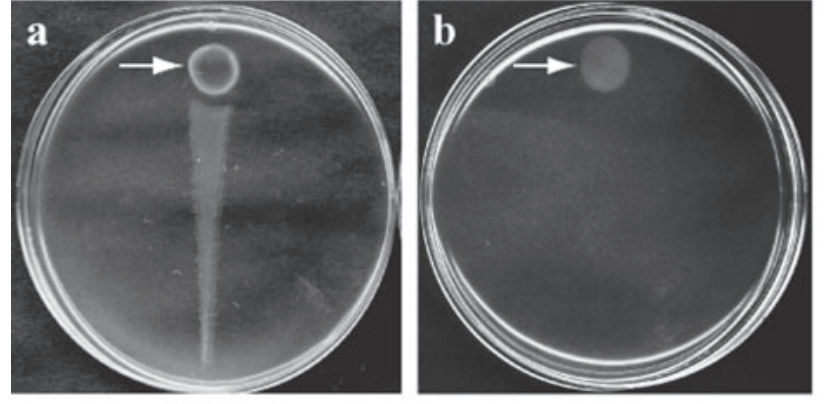

$\mathrm{S}$

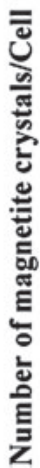

d

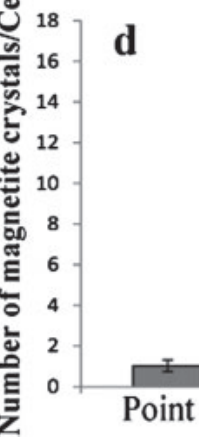

Point 1

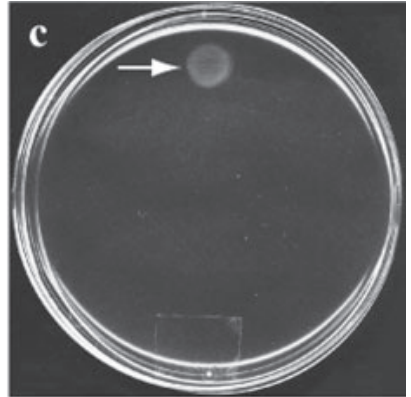

$\mathrm{S}$

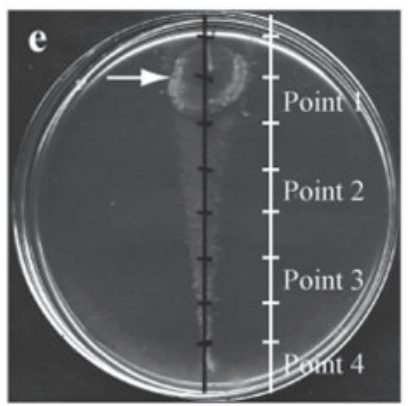

Fig. 1. Magnetic Swimming Assay of M. magneticum AMB-1.

M. magneticum AMB-1 WT cells were inoculated onto swimming plates with a neodymium magnet (a) and without one (b). Arrows show inoculation sites on the swim plates. A wedge-like halo formed on the plate with a magnet, and a circular halo formed on the plate without a magnet. Boxes indicate the positions of the south pole of the magnet. (c) Magnetic swimming assay of nonmagnetic cells. (d) Mean number of magnetite crystals per cell harvested from different positions in the wedge-like shaped halo. Sampling positions in the halo are shown in panel (e). Mean numbers of magnetite crystals were calculated by counting the numbers of electron dense crystals in at least 50 individual cells with a JEOL JEM 2000EX transmission electron microscope at an accelerating voltage of $120 \mathrm{kV}$ in bright-field mode.

$10 \mathrm{~min}$ at $25^{\circ} \mathrm{C}$. The numbers of magnetite crystals at several points in the cells were counted by TEM.

As shown Fig. 1d, the cells collected from point 1 contained only a small number of magnetite crystals $(1.0 \pm 0.3$ crystals/cell, $\mathrm{n}=50)$. This result indicates that the cells at point 1 were nonmagnetic. It has been reported that the culture of Magntosprillium contains small amounts of spontaneous nonmagnetic cells. ${ }^{27)}$ Such cells are probably attributable to the genetic instability of the magnetosome island. ${ }^{43}$ The spontaneous nonmagnetic cells in the culture could not migrate to the magnet, so they formed a circular halo at the inoculation site (Fig. 1e, arrow). The numbers of magnetite crystals per cell in the bacteria at points 2 , 3 , and 4 were $11.3 \pm 0.6$ crystals/cell $(n=50), 15.1 \pm$ 0.6 crystals/cell $(\mathrm{n}=50)$, and $16.2 \pm 0.7$ crystals $/$ cell $(\mathrm{n}=50)$ respectively (Fig. $1 \mathrm{~d})$.

These results indicate that the length of the swimming halo depended on the number of magnetite crystals per cell. Cells containing a sufficient number of magnetite crystals migrated from the inoculation site to the magnet along magnetic field lines, and then formed swimming halos at points 2,3 , and 4 .

Isolation of magnetotactic motility mutants using the magnetic swimming assay

Swimming assay is used to screen for motile and chemotactic mutants. ${ }^{40,44)}$ We used the magnetic swimming assay as a screening method to isolate magnetotactic motility mutants. We isolated two spontaneous mutants, mutant 1 and mutant 2 (Fig. 2).

The swimming halo of mutant 1 showed a circular shape (Fig. 2b), while mutant 1 had no magnetite crystals according to TEM observation (Fig. 2e). This result suggests that the circular halo of mutant 1 resulted from a defect in magnetite synthesis. The shape of the swimming halo of mutant 2 was different from that of WT (Fig. 2a, c). The swimming halo near the magnet followed a magnetic field line, while the swimming halo remote from the magnet was not oriented on a magnetic field line (Fig. 2c). According to TEM observation, the magnetite crystal size of mutant 2 was $23.7 \pm 1.5 \mathrm{~nm}$ $(\mathrm{n}=60)$ (Fig. 2f, h) and that of WT was $47.5 \pm 1.3 \mathrm{~nm}$ $(\mathrm{n}=73) \quad$ (Fig. 2d, g). M. magneticum AMB-1 WT synthesizes single domain paramagnetic magnetite crystals that range from 30 to $50 \mathrm{~nm}^{45,46)}$ On the other hand, mutant 2 synthesized the magnetite crystal of less than $30 \mathrm{~nm}$. That magnetite crystals was superparamagnetic. ${ }^{47,48)}$ It is likely that due to the superparamagnetic particle in the magnetosomes, mutant 2 showed magnetism only near the magnet in the swimming plate, and they did not orient to a magnetic field in remote areas from the magnet (Fig. 2c). The phenotypes of the two spontaneous mutants in the swimming plate were involved in magnetosome formation. This finding suggests that the magnetic swimming assay is applicable in screening mutants involving magnetotaxis.

Functional analysis of the MamK cytoskeleton by a magnetic swimming assay

If the magnetic swimming assay can be used to analyze the phenotypes of mutants of magnetosomeassociated proteins, it should prove useful to investigate the functions of magnetosome-associated proteins. We analyzed the phenotype of the mamK deletion strain by the swimming assay. MamK is a new member of the 

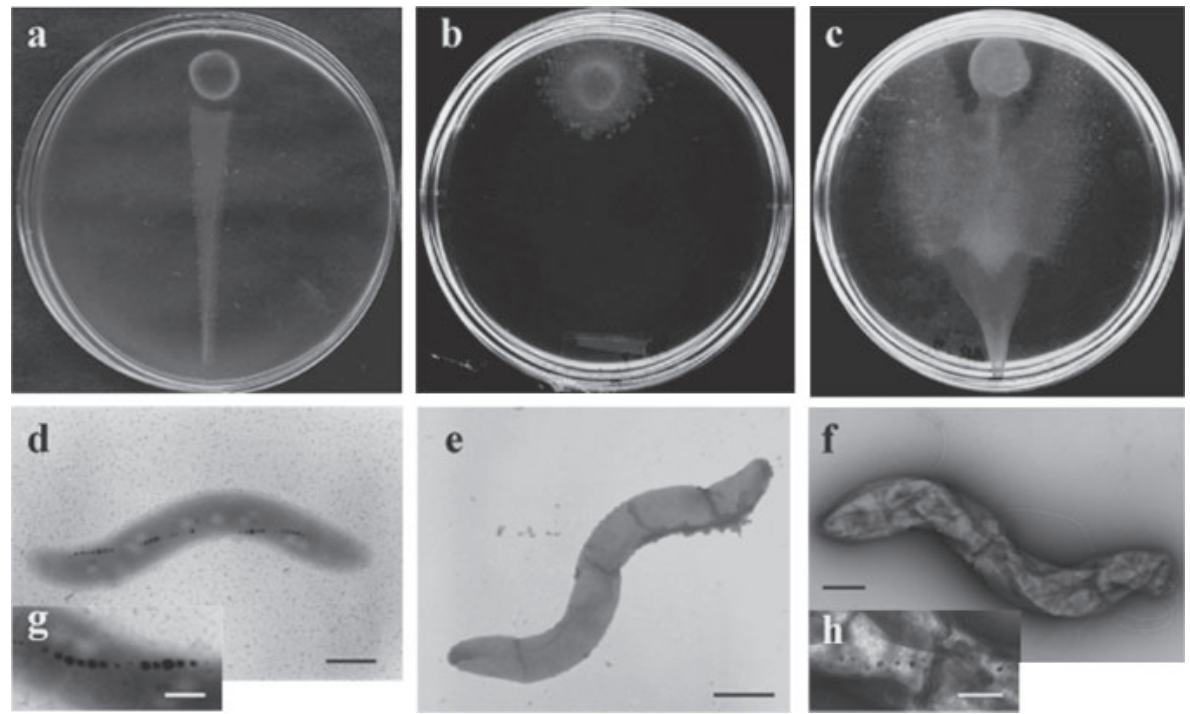

Fig. 2. Mutants Isolated Using the Magnetic Swimming Assay.

The magnetic swimming halo of WT (a), mutant 1 (b), and mutant 2 (c). Transmission electron micrographs of WT (d) mutant 1 (e) and mutant 2 (f). Scale bar is $500 \mathrm{~nm}$. The cells were negatively stained with $2 \%$ uranyl acetate. High magnification image of magnetosome chains of WT (g) and mutant 2 (h). Scale bar is $200 \mathrm{~nm}$. Mutant 2 cells had small magnetite crystals $(23.7 \pm 1.5 \mathrm{~nm}, \mathrm{n}=60)$. On the other hand, WT had normal size magnetite crystals $(47.5 \pm 1.3 \mathrm{~nm}, \mathrm{n}=73)$.
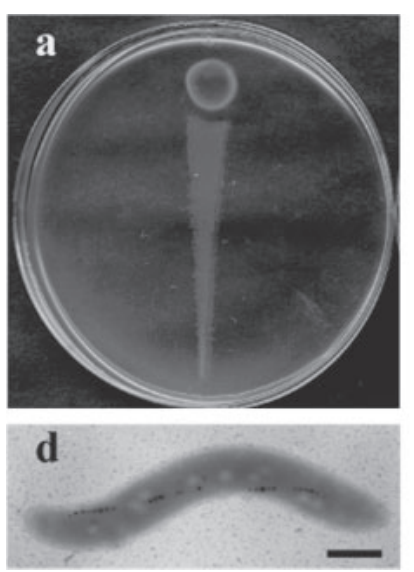
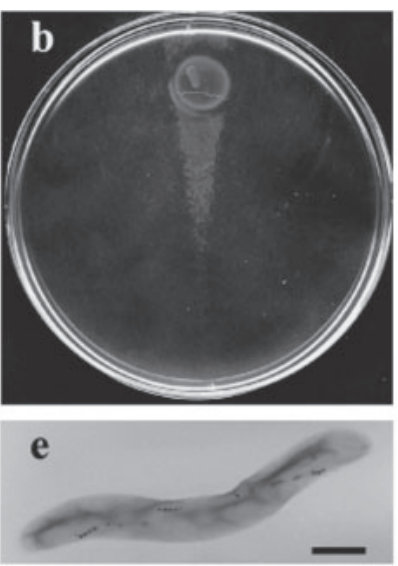
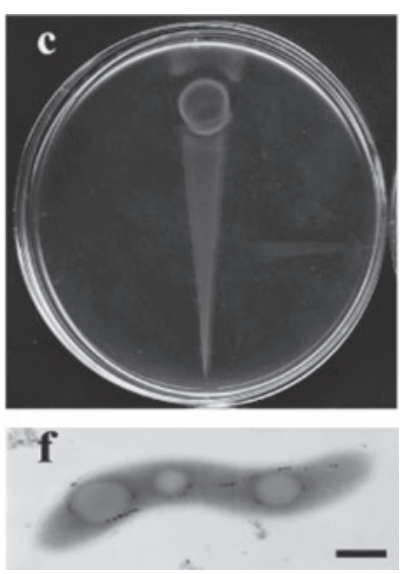

Fig. 3. Magnetic Swimming Assay of a $\Delta \operatorname{mam} K$ Strain.

Magnetic swimming halo of WT (a), $\triangle \operatorname{mam} K$ strain (b), and $\triangle m a m K$ pBBR-tac-mamK (complementary strain) (c). TEM images of WT (d), $\Delta m a m K$ strain (e), and $\Delta m a m K$ pBBR-tac-mamK (complementary strain) (f). Scale bar is $500 \mathrm{~nm}$.

bacterial actin homologs and is conserved specifically among magnetotactic bacteria. MamK polymerized in filamentous structure in vivo ${ }^{49)}$ and in vitro. ${ }^{50)}$ Komeili et al. found MamK filaments running parallel along magnetosome chains in a M. magneticum AMB-1 cell by electron cryotomography. ${ }^{26)}$ Moreover, they reported that in $\Delta m a m K$ cell, small groups of a few neighboring magnetosomes separated by large gaps appeared dispersed throughout the cell. They suggested that MamK is important in maintaining the magnetosome chain after the production of magnetosomes, but the details of the function of the MamK filaments remain unclear. To determine the function of MamK, we analyzed the phenotype of the mamK deletion strain by magnetic swimming assay.

Figure 3 shows the results of magnetic swimming assay of the $\Delta m a m K$ strain. The strain formed a wedgelike shaped halo similar to that of WT. The length of the swimming halo of the $\Delta m a m K$ strain was shorter than that of WT (Fig. 3a, b), in that the length of the swimming halo of $\triangle m a m K$ was approximately $70 \%$ that of the WT (Fig. 4a). The expression and localization of MamK in the mamK complementary strain were confirmed by immunoblotting (Supplemental Fig. S2a) and immunofluorescence (Supplemental Fig. S2b). The length of the swimming halo of the complementary strain was restored to that of WT (Fig. 3c). These results indicate that the magnetic swimming assay showed differences between $\triangle m a m K$ and WT, even though there were no significant differences in the $C_{\text {mag }}$ values (Table 2) or the TEM observations (Fig. 3d, e) between $\triangle m a m K$ or WT. In addition, these strains had similar swimming speeds in liquid medium and had similar sizes of magnetite crystals (Table 2). These results clearly indicate that the magnetic swimming assay is useful in investigating the function of magnetosomeassociated proteins in magnetotaxis.

Effects of iron concentration on the swimming halos of the $\Delta$ mamK strain

There were no differences in magnetism $\left(C_{m a g}\right)$, swimming speeds, or sizes of magnetite crystals as 
Table 2. Measurements of Magnetism, Swimming Speeds and the Size of Magnetite Crystals for Each Strain

\begin{tabular}{|c|c|c|c|c|}
\hline $\begin{array}{c}\text { Strains } \\
\text { (M. magneticum AMB-1) }\end{array}$ & $\mathrm{OD}_{600}{ }^{\mathrm{a}}$ & $C_{m a g}$ values $^{\mathrm{b}}$ & $\begin{array}{l}\text { Swimming speeds } \\
(\mu \mathrm{m} / \mathrm{s})^{\mathrm{c}}\end{array}$ & $\begin{array}{c}\text { The size of magnetite } \\
\text { crystals }(\mathrm{nm})^{\mathrm{d}}\end{array}$ \\
\hline WT & $0.103 \pm 0.003$ & $1.08 \pm 0.040$ & $71.9 \pm 1.9$ & $47.5 \pm 1.3$ \\
\hline WT pBBR-tac & $0.100 \pm 0.004$ & $1.04 \pm 0.036$ & - & - \\
\hline WT pBBR-tac-mamK & $0.101 \pm 0.008$ & $1.12 \pm 0.026$ & - & - \\
\hline$\Delta m a m K$ & $0.098 \pm 0.002$ & $1.07 \pm 0.023$ & $73.7 \pm 2.1$ & $46.6 \pm 1.2$ \\
\hline$\Delta m a m K$ pBBR-tac & $0.108 \pm 0.007$ & $1.06 \pm 0.031$ & - & - \\
\hline$\Delta m a m K$ pBBR-tac-mamK & $0.104 \pm 0.001$ & $1.06 \pm 0.034$ & $73.1 \pm 2.5$ & $46.2 \pm 1.4$ \\
\hline
\end{tabular}

${ }^{a}$ Optical density at $600 \mathrm{~nm}$. Results represent means for three independent experiments.

${ }^{\mathrm{b}}$ The magnetism of cells was defined as $\left(\mathrm{OD}_{\max } / \mathrm{OD}_{\min }\right)-1$. Results represent means for three independent experiments.

${ }^{\mathrm{c}}$ Swimming speeds of cells in liquid culture. Results shown are means for swimming speeds, for which 50 independent cells were measured.

${ }^{\mathrm{d}}$ Sizes of magnetite crystals in cells. The results shown are mean sizes of magnetite crystals, for which at least 100 particles were measured.

a

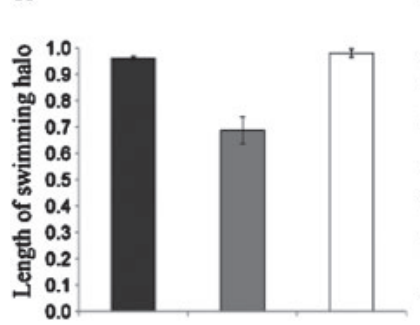

b

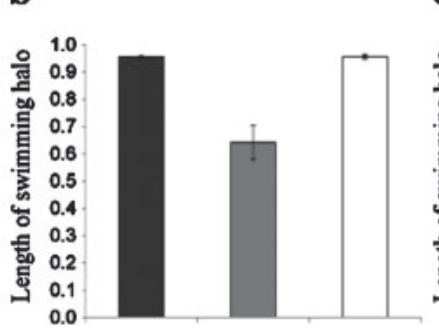

c

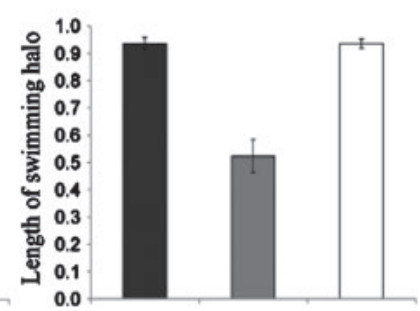

Fig. 4. Effects of Various Iron Concentrations on Lengths of Swimming Halos.

Each strain of $M$. magneticum AMB-1 was inoculated onto a magnetic swim plate at an iron concentration of $35 \mu \mathrm{M}(\mathrm{a}), 18 \mu \mathrm{M}(\mathrm{b})$, or $9 \mu \mathrm{M}(\mathrm{c})$. Data show the mean length of the swimming halo of the WT (black bar), $\Delta m a m K$ (gray bar), and $\Delta m a m K$ pBBR-tac-mamK (white bar). The mean length of the swimming halo was scored as the distance of the swimming halo for each strain (mm) divided by the distance of the inoculated cell site from the magnet $(\mathrm{mm})$. Fifteen swim plate experiments were repeated 3 times for each strain. Error bars represent standard error.

between WT and $\Delta m a m K$ strain (Table 2). However, the length of the swimming halo was significantly different as between WT and $\triangle m a m K$ strain. To determine the reason for this difference, we conducted the magnetic swimming assay at different iron concentrations. The numbers of magnetite crystals decreased in the magnetotactic bacteria with decreasing iron concentrations in the medium. ${ }^{51)} \mathrm{We}$ examined to determine whether that the length of swimming halo would be affected by decreasing the numbers of magnetite crystals in a cell. The WT and the $\Delta m a m K$ strains were inoculated onto the swim plates at iron concentrations of $35 \mu \mathrm{M}, 18 \mu \mathrm{M}$, and $9 \mu \mathrm{M}$. The swim plates were incubated at $28^{\circ} \mathrm{C}$ under a $1 \% \mathrm{O}_{2}-99 \% \mathrm{~N}_{2}$ atmosphere in the dark. After incubation for $7 \mathrm{~d}$, we calculated the mean length of the swimming halos.

The length of the swimming halo of the WT was the same at iron concentrations of $35 \mu \mathrm{M}, 18 \mu \mathrm{M}$, and $9 \mu \mathrm{M}$ (Fig. 4). On the other hand, the length of the swimming halo of the $\Delta m a m K$ strain was $72 \%, 67 \%$, and $55 \%$ that of the WT at iron concentrations of $35 \mu \mathrm{M}, 18 \mu \mathrm{M}$, and $9 \mu \mathrm{M}$ respectively (Fig. 4). The length of the swimming halo of the complementary strain was the same as that of the WT at each iron concentration (Fig. 4). The length of the swimming halo of the $\Delta m a m K$ strain was progressively shorter with decreasing iron concentrations in the swim plate. According to Fig. 1d, the length of the swimming halo depended on the numbers of magnetite crystals in cell. These results suggest that the numbers of magnetite crystals in the $\Delta$ mamK cells decreased. Therefore, the shorter swimming halo in $\Delta m a m K$ was due to a decreased numbers of magnetite crystals in the cells.
Numbers of magnetite crystals in $\Delta$ mamK cells at the mid-log phase and the early stationary phase

We hypothesized that the shorter swimming halo for $\triangle m a m K$ was due to a decreased numbers of magnetite crystals in the cells. To test this, we counted the numbers of magnetite crystals in the cells by TEM. The WT, $\triangle m a m K$, and complementary strains were cultured at $28^{\circ} \mathrm{C}$ for $24 \mathrm{~h}$ (mid-log phase) and $48 \mathrm{~h}$ (early stationary phase) in EMSGM at different iron concentrations (Table 3). The numbers of magnetite crystals in WT cells were not affected by the iron concentration in the media in the mid-log $(24 \mathrm{~h})$ or the early stationary $(48 \mathrm{~h})$ phase (Table 3 ). The WT cells contained 12-14 magnetite crystals at the mid-log phase, while the WT cells contained 16-18 magnetite crystals at the early stationary phase. The numbers of magnetite crystals in the cells were constant at each iron concentration of the media. In the mid-log phase $(24 \mathrm{~h})$ and the early stationary phase $(48 \mathrm{~h})$, the numbers of magnetite crystals in the $\Delta m a m K$ cells were almost constant (710 particles for $24 \mathrm{~h}, 15-17$ particles for $48 \mathrm{~h}$ ) at each iron concentration. The numbers of magnetite crystals of the $\Delta$ mamK cells at the mid-log phase $(24 \mathrm{~h})$ were significantly lower than that of the WT cells, while the numbers of magnetite crystals in the complementary strain were similar to that in WT at both the mid-log phase $(24 \mathrm{~h})$ and the early stationary phase $(48 \mathrm{~h})$.

We checked the distribution of the numbers of magnetite crystals in these strains by TEM observation (Fig. 5). Although the distribution of the numbers of magnetite crystals in the $\Delta m a m K$ cells was similar to that of the WT cells at the early stationary phase $(48 \mathrm{~h})$, that of the $\Delta m a m K$ cells at the mid-log phase $(24 \mathrm{~h})$ 
Table 3. Mean Numbers of Magnetite Crystals per Cells at Each Iron Concentration at Various Growth Phases

\begin{tabular}{|c|c|c|c|c|c|c|}
\hline \multirow{3}{*}{ Strains } & \multicolumn{6}{|c|}{ Number of magnetite crystals (crystals/cell) ${ }^{\mathrm{a}}$} \\
\hline & \multicolumn{3}{|c|}{ The mid-log phase $(24 \mathrm{~h})^{\mathrm{b}}$} & \multicolumn{3}{|c|}{ The early stationary phase $(48 \mathrm{~h})^{\mathrm{b}}$} \\
\hline & $\mathrm{Fe} 35 \mu \mathrm{M}^{\mathrm{c}}$ & $\mathrm{Fe} 18 \mu \mathrm{M}^{\mathrm{c}}$ & $\mathrm{Fe} 9 \mu \mathrm{M}^{\mathrm{c}}$ & $\mathrm{Fe} 35 \mu \mathrm{M}^{\mathrm{c}}$ & $\mathrm{Fe} 18 \mu \mathrm{M}^{\mathrm{c}}$ & $\mathrm{Fe} 9 \mu \mathrm{M}^{\mathrm{c}}$ \\
\hline WT & $13.8 \pm 0.7$ & $13.6 \pm 0.6$ & $13.0 \pm 0.5$ & $17.6 \pm 0.6$ & $17.2 \pm 0.6$ & $17.3 \pm 0.5$ \\
\hline$\Delta m a m K$ & $9.5 \pm 0.9$ & $8.9 \pm 0.8$ & $8.1 \pm 0.9$ & $16.4 \pm 0.7$ & $16.0 \pm 0.7$ & $15.5 \pm 0.7$ \\
\hline$\Delta m a m K$ pBBR-tac-mamK & $13.9 \pm 0.8$ & $13.8 \pm 0.7$ & $12.9 \pm 0.8$ & $17.1 \pm 0.6$ & $17.0 \pm 0.6$ & $17.0 \pm 0.5$ \\
\hline
\end{tabular}

${ }^{a}$ The mean numbers of magnetite crystals in cells, for which at least 50 cells was measured.

${ }^{\mathrm{b}}$ Growth phase of each strain of cells.

${ }^{\mathrm{c}}$ Iron concentration of the cultures.
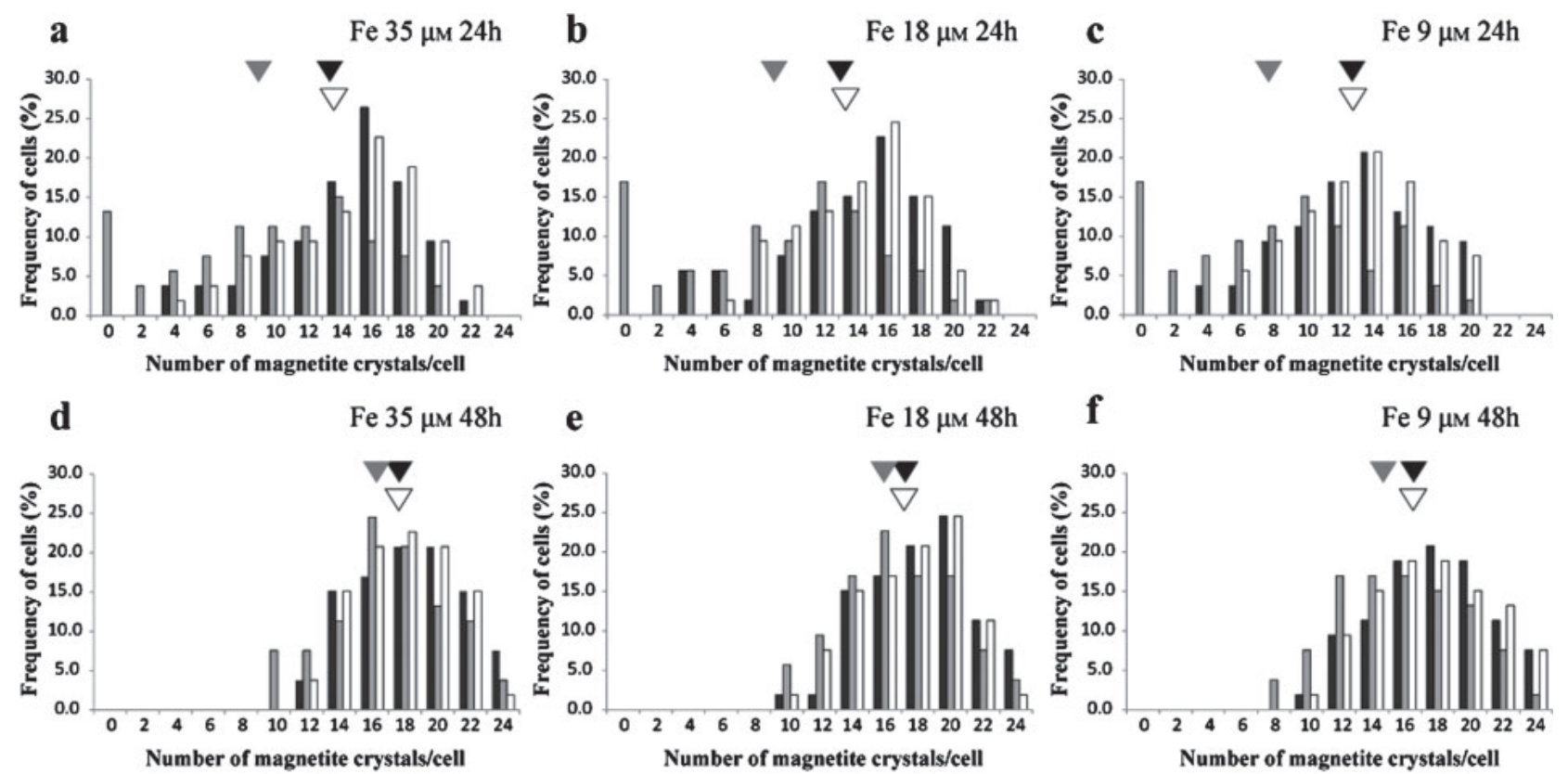

Fig. 5. Effects of Incubation Time on the Numbers of Magnetite Crystals of Each Strain at Various Iron Concentrations.

Each M. magneticum AMB-1 strain was inoculated into liquid EMSGM at various iron concentrations and incubated to the mid-log phase $(24 \mathrm{~h})$ or the early stationary phase $(48 \mathrm{~h})$. Histogram showing the numbers of magnetite crystals in WT (black bar), $\Delta m a m K$ (gray bar), and $\triangle m a m K$ pBBR-tac-mamK (white bar). These strains were inoculated into liquid EMSGM at iron concentrations of $35 \mu \mathrm{M}$ (a), $18 \mu \mathrm{M}$ (b), and $9 \mu \mathrm{M}$ (c) for an incubation time of $24 \mathrm{~h}$, and of $35 \mu \mathrm{M}(\mathrm{d}), 18 \mu \mathrm{M}(\mathrm{e})$, and $9 \mu \mathrm{M}$ (f) for an incubation time of $48 \mathrm{~h}$. The numbers of magnetite crystals per cell was counted in at least 50 cells of each strain under TEM observation. Triangle shows the mean numbers of magnetite crystals in WT (black), $\Delta m a m K$ (gray), and $\triangle m a m K$ pBBR-tac-mamK (white) respectively (Table 3 ).

differed from that of the WT. Notably, $13-17 \%$ of the $\Delta m a m K$ cells had no magnetite crystals at the mid-log phase $(24 \mathrm{~h})$ at each iron concentrations (Fig. 5a, b, c). The distribution of the numbers of magnetite crystals in the complementary strain cells was same as that in WT (Fig. 5).

These results indicate that the numbers of magnetite crystals in the $\triangle \operatorname{mamK}$ cells decreased at the mid-log phase (Fig. 5, Table 3). The length of the swimming halo of the $\Delta m a m K$ strain was shorter than that of WT (Figs. 3 and 4). The length of the swimming halo depended on the numbers of magnetite crystals in the cells (Fig. 1d). Hence, the shorter swimming halo of $\Delta m a m K$ strain was due to the lower numbers of magnetite crystals per cell at the mid-log phase. During magnetic swimming assay, magnetotactic bacteria grew continuously in the semi-solid medium plate and formed a swimming halo, suggesting that the cells grew logistically during this assay.

A previous study of the $\Delta m a m K$ strain indicated that MamK is dynamically involved in the positioning and assembly of the magnetosome chain. ${ }^{52,53)}$ Recently,
Katzmann et al. suggested that the MamK filament is required for proper magnetosome chain positioning and segregation in Magnetospillium gryphiswaldense MSR$1 .^{54,55)}$ Our results indicate that some $\Delta \operatorname{mamK}$ cells had no magnetite crystals at the mid-log phase (Fig. 5). The lack of magnetite crystals in the $\Delta \operatorname{mamK}$ cells was due to the absence of the MamK filament. Thus, MamK filaments might be required to segregate magnetosomes to daughter cells. Because the $\Delta m a m K$ cells did not segregate the magnetosomes to the daughter cells, some of daughter cells did not orient magnetic field line. Hence the length of the swimming halo of the $\Delta m a m K$ strains was shorter than that of the WT.

In the magnetic swimming assay, the length of the swimming halo indicated the numbers of magnetite crystals in a cell. In this study, we found that this method can assess the magnetism in magnetotaxis. On the other hand, the swimming assay can assess the bacterial motility based on the elongation of the swimming halo. Accordingly, in the magnetic swimming assay, the wedge-shaped halo can be formed by magnetism and by motility. Therefore, the magnetic swimming assay can 
assess magnnetotaxis. By the magnetic swimming assay, it might be possible to isolate magnetotactic motility mutants. Hence the assay is a useful tool to ascertain magnetotaxis.

\section{Conclusions}

In the present study, we established a qualitative method of assessing the magnetotactic motility of magnetotactic bacteria. This method is based on a bacterial swimming assay with a magnet. In this method, magnetotactic motility is represented as the length of the swimming halo. We named the method the magnetic swimming assay. Although the conventional method $\left(C_{\text {mag }}\right)$ does not distinguish between the WT and the $\triangle m a m K$ strain, the magnetic swimming assay can be used to assess differences in these strains based on the lengths of swimming halos. These findings clearly demonstrated distinct advantages of the magnetic swimming assay for analyzing magnetotaxis mutant and magnetosome-associated proteins in studying the functions of these proteins in magnetotactic motility.

The magnetic swimming assay should be useful for assessing magnetotaxis by comparing the swimming halos of the deletion mutants of individual genes involved in the magnetosome island. In addition, this it is applicable to the screening of magnetotaxis mutants and can thus be used to study bacterial magnetotaxis for potential utilization of magnetosomes.

\section{Acknowledgments}

We thank Dr. Arash Komeili (University of California, Berkeley, CA) for providing the mamK deletion strain of M. magneticum AMB-1. This work was supported by Grant-in-Aid for Scientific Research on Innovative Areas (Grants nos. 24117007 and 23111508) and a Grant-in-Aid for Young Scientists (B) (Grant no. 22780063) from the Ministry of Education, Culture, Sports, Science, and Technology and Japan Society for the Promotion of Science.

\section{References}

1) Bazylinski DA and Frankel RB, Nat. Rev. Microbiol., 2, 217 230 (2004)

2) Faivre D and Schüler D, Chem. Rev., 108, 4875-4898 (2008).

3) Komeili A, Апnи. Rev. Biochem., 76, 351-366 (2007).

4) Komeili A, FEMS Microbiol. Rev., 36, 232-255 (2011).

5) Schüler D, FEMS Microbiol. Rev., 32, 654-672 (2008).

6) Blakemore RP, Frankel RB, and Kalmijn AJ, Nature, 286, 384 385 (1980).

7) Frankel RB, Bazylinski DA, Johnson MS, and Taylor BL, Biophys. J., 73, 994-1000 (1997).

8) Bahaj AS and James PAB, IEEE Trans. Magn., 29, 3358-3360 (1993).

9) Seong S and Park HT, Biotechnol. Bioeng., 76, 11-16 (2001).

10) Steinberger B, Petermann H, and Weiss DG, J. Fluid Mech., 273, 189-211 (1994).

11) Rosenblatt C, Torres de Araujo FF, and Frankel RB, J. Appl. Phys., 53, 2727-2729 (1982).

12) Rosenblatt C, Torres de Araujo FF, and Frankel RB, Biophys. J., 40, 83-85 (1982)

13) Schüler D, Uhl R, and Bauerlein E, FEMS Microbiol. Lett., 132, 139-145 (1995).

14) Zhao LZ, Wu D, Wu LF, and Song T, J. Biochem. Biophys. Method., 70, 363-368 (2007).

15) Matsunaga T, Okamura $Y$, Fukuda $Y$, Wahyudi AT, Murase $Y$, and Takeyama H, DNA Res., 12, 157-166 (2005).

16) Richter $M$, Kube $M$, Bazylinski DA, Lombardot $T$, and Glockner FO, J. Bacteriol., 189, 4899-4910 (2007).

17) Ullrich S, Kube M, Schübbe S, Reinhardt R, and Schüler D, J. Bacteriol., 187, 7176-7184 (2005).

18) Bazylinski DA and Schübbe S, Adv. Appl. Microbiol., 62, 21-62 (2007).

19) Fukuda $\mathrm{Y}$, Okamura $\mathrm{Y}$, Takeyama $\mathrm{H}$, and Matsunaga $\mathrm{T}$, FEBS Lett., 580, 801-812 (2006).

20) Schübbe S, Kube M, Scheffel A, Wawer C, and Heyen U, J. Bacteriol., 185, 5779-5790 (2003).

21) Scheffel A and Schüler D, J. Bacteriol., 189, 6437-6446 (2007).

22) Scheffel A, Gruska M, Faivre D, Linaroudis A, Plitzko JM, and Schüler D, Nature, 440, 110-114 (2006).

23) Scheffel A, Gärdes A, Grünberg K, Wanner G, and Schüler D, J. Bacteriol., 190, 377-386 (2008).

24) Murat D, Quinlan A, Vali H, and Komeili A, Proc. Natl. Acad. Sci. USA, 107, 5593-5598 (2010).

25) Blakemore RP, Science, 190, 377-379 (1975).

26) Komeili A, Li Z, Newman DK, and Jensen GJ, Science, 311, 242-245 (2006).

27) Blakemore RP, Maratea D, and Wolfe RS, J. Bacteriol., 140, 720-729 (1979).

28) Jerpseth B, Greener A, Short JM, Viola J, and Kretz PL, Mol. Biol., 5, 81-83 (1992).

29) Demarre G, Guérout AM, Chiho M, Rowe-Magnus DA, Marlière P, and Mazel D, Res. Microbiol., 156, 245-255 (2005).

30) Li JH, Pan YX, Chen GJ, Liu QS, Tian LX, and Lin W, Geophys. J. Int., 177, 33-42 (2009).

31) Amann E, Brosius J, and Ptashne M, Gene, 25, 167-178 (1983).

32) Alting-Mees MA and Short JM, Nucleic Acids Res., 17, 9494 (1989).

33) Newman JR and Fuque C, Gene, 277, 197-203 (1999).

34) Komeili A, Vali H, Beveridge TJ, and Newman DK, Proc. Natl. Acad. Sci. USA, 101, 3839-3844 (2004).

35) Yang C, Takeyama H, Tanaka T, and Matsunaga T, Enzyme Microb. Technol., 29, 13-19 (2001).

36) Adler J, Science, 153, 708-716 (1966).

37) Fraser GM and Hughes C, Curr. Opin. Microbiol., 2, 630-635 (1999).

38) Harshey RM, Аnnu. Rev. Microbiol., 57, 249-273 (2003).

39) Turner L, Zhang R, Darnton NC, and Berg HC, J. Bacteriol., 192, 3259-3267 (2010).

40) Ito M, Terahara N, Fujinami S, and Krulwich TA, J. Mol. Biol., 352, 396-408 (2005).

41) Shinoda S and Okamoto K, J. Bacteriol., 129, 1266-1271 (1977).

42) Rashid MH and Kornberg A, Proc. Natl. Acad. Sci. USA, 97, 4885-4890 (2000).

43) Ullrich S, Kube M, Schübbe S, Reinhardt R, and Schüler D, J. Bacteriol., 187, 7176-7184 (2005).

44) Hazelbauer GL, Mesibov RE, and Adler J, Proc. Natl. Acad. Sci. USA, 64, 1300-1307 (1969).

45) Arakaki A, Webb J, and Matsunaga T, J. Biol. Chem., 278, 8745-8750 (2003).

46) Lins U, Farina M, and Kachar B, Microbiol. Res., 158, 317-320 (2003).

47) Butler RF and Subir KB, J. Geophys. Res., 80, 252-259 (1975).

48) Robert EK and Kirsch JL, Earth-Sci. Rev., 86, 42-61 (2008).

49) Pradel N, Santini CL, Bernadac A, Fukumori Y, and Wu LF, Proc. Natl. Acad. Sci. USA, 103, 17485-17489 (2006).

50) Taoka A, Asada R, Wu LF, and Fukumori Y, J. Bacteriol., 189, 8737-8740 (2007).

51) Schüler D and Baeuerlein E, Arch. Microbiol., 166, 301-307 (1996).

52) Scheffel A, Gruska M, Faivre D, Linaroudis A, Plitzko JM, and Schüler D, Nature, 440, 110-114 (2006).

53) Komeili A, Аnпи. Rev. Biochem., 76, 351-366 (2007).

54) Katzmann E, Müller F, Lang C, Messerer M, Winklhofer M, Plitzko JM, and Schüler D, Mol. Microbiol., 82, 1316-1329 (2011).

55) Katzmann E, Scheffel A, Gruska M, Plitzko JM, and Schüler D, Mol. Microbiol., 77, 208-224 (2010). 